\title{
Alternsforschung
}

\section{Der Türkise Prachtgrundkärpfling - ein Leben im Zeitraffer}

JOHANNES KRUG, ANNEKATRIN RICHTER, HANNA REUTER, CHRISTOPH ENGLERT LEIBNIZ-INSTITUT FÜR ALTERNSFORSCHUNG - FRITZ-LIPMANN-INSTITUT (FLI), JENA

\section{Nothobranchius furzeri is a killifish from southeast Africa with an extremely short lifespan. Aging of this fish shows significant similari- ties to the aging process in mammals including humans. N. furzeri is thus an attractive model organism for scientists in the field of aging research. Here, we present some key aspects of the biology of the killi- fish and discuss how this fish can help to better understand aging and aging-related diseases.}

DOI: $10.1007 / \mathrm{s} 12268-020-1393-3$

(C) Die Autoren 2020

Altern ist ein kontinuierlicher, nicht umkehrbarer biologischer Prozess, der ab einem bestimmten Zeitpunkt mit der Abnahme von Organfunktionen verbunden ist. Als Konsequenz steigen die Krankheitsanfälligkeit und Sterbewahrscheinlichkeit mit dem chronologischen Alter. Angesichts der alternden Bevölkerung sind altersassoziierte Krankheiten eine Herausforderung für die heutige und zukünftige Gesellschaft. Die Erforschung von Alternsprozessen rückt deshalb immer mehr in den Fokus der Wissenschaft. Die Wahl geeigneter Modellsysteme ist dabei von großer Bedeutung. Die Fliege Drosophila melanogaster oder der Fadenwurm Caenorhabditis elegans sind mit ihren kurzen Lebensspannen seit Jahren etablierte Modellorganismen zur Erforschung des Alterns. Allerdings besitzen sie eine erhebliche evolutionäre Distanz zum Menschen. Die Maus andererseits ist mit dem Menschen näher verwandt, ihre Lebensspanne von bis zu 3 Jahren macht aber entsprechende Forschungsprojekte langwierig. Der Türkise Prachtgrundkärpfling (Nothobranchius furzeri) ist das Wirbeltier mit der kürzesten Lebensspanne, das zurzeit im Labor gehalten werden kann [1]. Damit ist er ein optimales Forschungsobjekt, um Alterungsprozessen auf den Grund zu gehen.

\section{Der Türkise Prachtgrundkärpfling -} live fast, die old

Der Türkise Prachtgrundkärpfling ist ein bis zu sechs Zentimeter großer Fisch, der zu den
Eierlegenden Zahnkarpfen (auch Killifische) gehört. Seine Verbreitung beschränkt sich auf saisonale Gewässer in Mosambik und Simbabwe, wo er 1968 im Gonarezhou-Nationalpark entdeckt wurde. Es existieren verschiedene Stämme, die sich zum einen in der Farbe ihrer Schwanzflosse und zum anderen in ihrer mittleren Lebenserwartung von drei bis sieben Monaten unterscheiden (Abb. 1A, [2]).

Die kurze Lebensspanne des Türkisen Prachtgrundkärpflings wird als Adaptation an die harschen Lebensbedingungen in den saisonalen Tümpeln in Südostafrika angesehen. In der Trockenperiode trocknen die Gewässer aus. Die Eier jedoch können, geschützt durch eine harte Eischale, im Bodengrund für lange Zeit überstehen und sich dort ohne Wasser weiterentwickeln. Dabei kann der Killifisch bis zu drei Diapausen durchlaufen. Dies sind Phasen, in denen die Entwicklung angehalten wird. Aus entwicklungsbiologischer Sicht sind Diapausen faszinierend. Abgesehen davon, dass Temperatur und Licht den Ein- und Austritt in bzw. aus der Diapause initiieren können, wissen wir unter anderem über die Regulation des Stoffwechsels und die Genaktivität in der Diapause sehr wenig. Sobald die Tümpel in der Regenzeit wieder mit Wasser gefüllt werden, beginnen die Fischlarven aus den Eiern zu schlüpfen. Während die Entwicklung im Ei über Monate bis sogar Jahre hinausgezögert werden kann, um lang anhal- tende Trockenphasen zu überstehen, beginnt nach dem Schlupf der Wettlauf gegen die Zeit. Die Schlüpflinge nehmen rasch an Gewicht und Größe zu, erreichen schon nach ungefähr zwei Wochen die Geschlechtsreife und altern für ein Wirbeltier vergleichsweise schnell (Abb. 1B, [3]).

\section{Afrika im Labor - ein Killifisch als Modellorganismus}

Trotz seiner kurzen Lebensspanne zeigt der Türkise Prachtgrundkärpfling typische Anzeichen des Alterns (siehe unten), weswegen er sich als Modellorganismus in der Alternsforschung etabliert hat. Bei Hobbyaquarianern ist der Killifisch aufgrund seiner Farbenpracht schon seit längerer Zeit sehr beliebt. Die erfolgreiche Haltung und insbesondere Vermehrung dieser Fischspezies sind jedoch wegen der Besonderheiten seiner Entwicklung nicht trivial.

Auch unter optimalen und stabilen Haltungsbedingungen mit ausreichend Futter bleiben besondere Merkmale wie das schnelle Erreichen der Geschlechtsreife sowie die kurze Generationszeit erhalten. Für eine erfolgreiche Haltung und Zucht dieser Fischart im Labor wurde eine Reihe von Verfahren entwickelt, welche die natürlichen Bedingungen in Afrika imitieren. So wird den Fischen im Labor eine Sandschale zur Eiablage in das Becken gestellt. Um anschließend die Trockenphase nachzustellen, werden die Eier auf eine Petrischale mit Kokosfasersubstrat überführt. Auf diesen Platten können die Eier für bis zu zwei Jahre in einem Inkubator gelagert werden, wobei sie ihre Entwicklung in einer der drei Diapausen arretieren können (Abb. 1C). Die Geschwindigkeit der Entwicklung kann durch die Aufbewahrungstemperatur $\left(25-29{ }^{\circ} \mathrm{C}\right)$ beeinflusst werden. Nach dem Umsetzen eines solchen Eis vom Kokosfasersubstrat ins Wasser schlüpft innerhalb kürzester Zeit die Fischlarve. Die Möglichkeit, den Schlupfbeginn derart zu beeinflussen, stellt einen enormen Vorteil für Arbeiten mit synchronisierten Populationen dar. Aufgrund ihres teilweise aggressiven Sozialverhaltens werden besonders adulte Männchen 
meist einzeln oder zusammen mit Weibchen in kleinen Zuchtgruppen gehalten. In freier Wildbahn ernähren sich die Fische in der Regel von Invertebraten, kleinen Krebstierchen und Insektenlarven. In der Laborhaltung muss berücksichtigt werden, dass die Fische nur Lebendfutter aufnehmen und sich nur schwer an herkömmliches Staub- oder Trockenfutter gewöhnen lassen. Aus diesem Grund werden Jungfische bis zu einem Alter von ca. fünf Wochen zweimal täglich mit Salzwasserkrebsen und danach einmal täg- lich mit frischen roten Mückenlarven gefüttert. In den letzten Jahren wurden Protokolle für die Laborhaltung dieser Fischspezies veröffentlicht, um einerseits die Haltungsbedingungen zu standardisieren und andererseits die Verbreitung des Türkisen Prachtgrundkärpflings als Modellorganismus in der Forschung voranzutreiben [4].

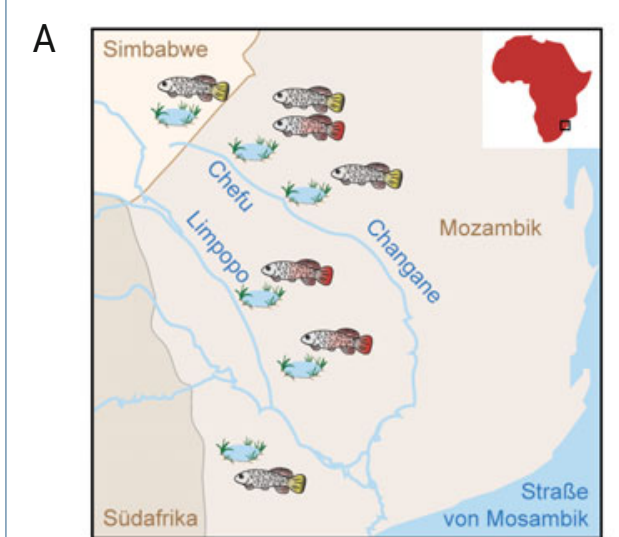

B
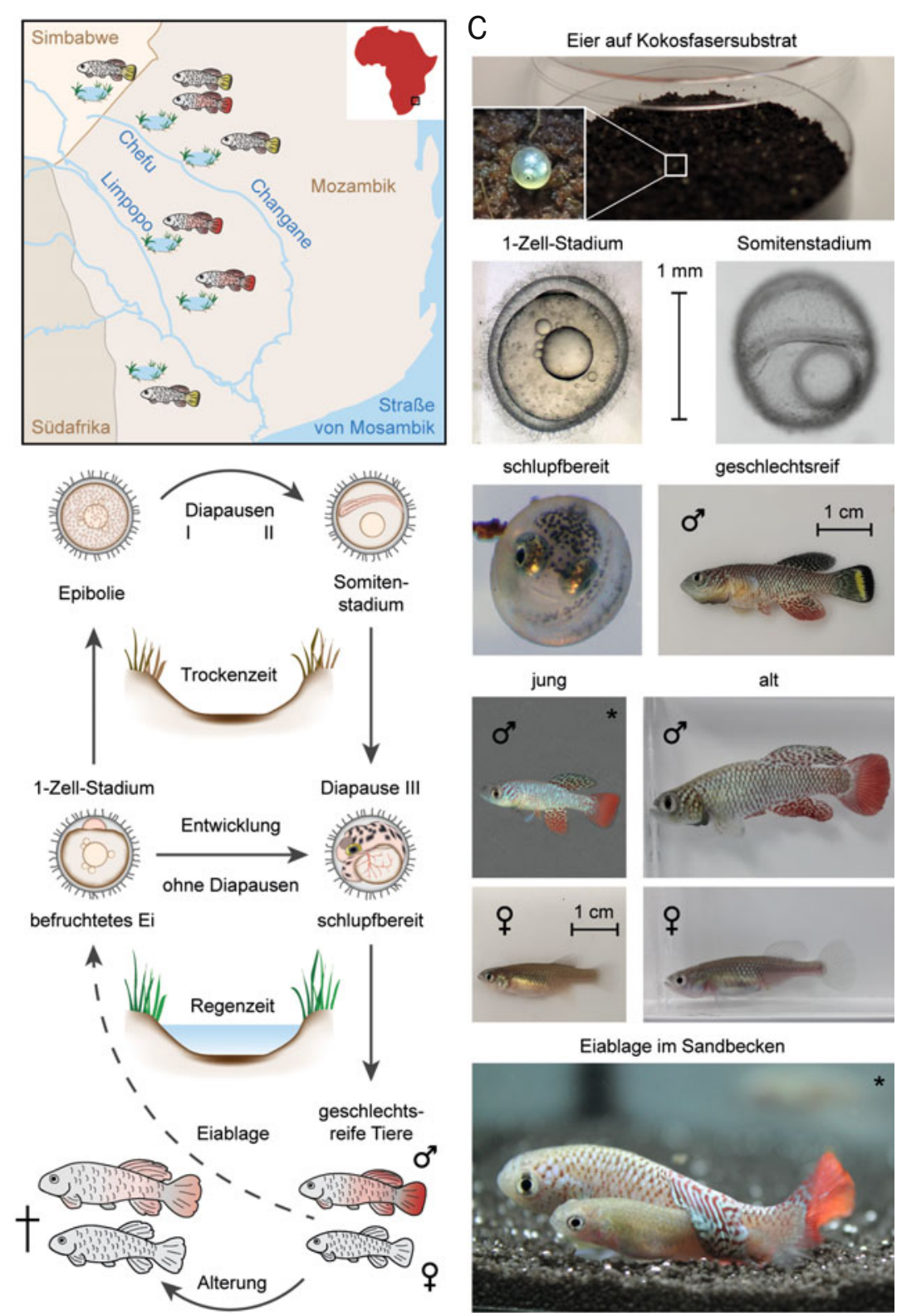

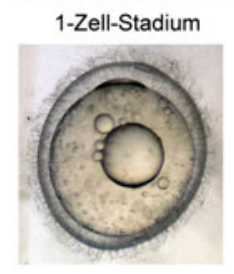

schlupfbereit

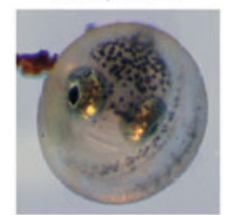

jung
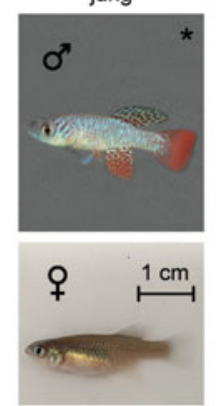

Eiablage im Sandbecken

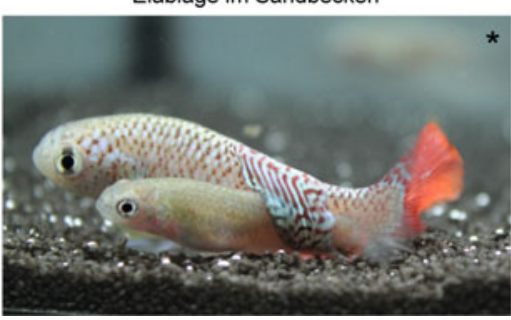

$\Delta$ Abb. 1: Afrika im Labor. A, Regionales Auftreten saisonaler Tümpel mit gelb- oder rotflossigen Türkisen Prachtgrundkärpflingen in Südostafrika. B, Im Lebenszyklus des Killifisches erlauben fakultative Diapausen in drei Embryonalstadien (Epibolie, Somiten- und schlupfbereites Stadium) das Überleben der Eier während der Trockenzeit. In der Regenzeit schlüpfen die Fische, wachsen heran und pflanzen sich fort. Die im Teichboden abgelegten Eier können sich alternativ auch in derselben Saison bis zum Schlupf entwickeln. C, Laborbeispiele. *Fotos: Nils Hartmann.

\section{Mithilfe des Killifisches}

\section{Alterungsprozesse besser verstehen}

Der Prachtgrundkärpfling zeigt typische äußere Alternserscheinungen, wie den Verlust von Pigmentierung, die Krümmung der Wirbelsäule und den Schwund an Muskelmasse. Weiterhin reduzieren sich Fertilität, Schwimmaktivität und die kognitive Leistungsfähigkeit [2]. Auch hinsichtlich molekularer Marker ähnelt das Altern des Fisches dem von Säugetieren. So zeigen ältere Prachtgrundkärpflinge eine erhöhte Seneszenz-assoziierte $\beta$-Galactosidase-Aktivität sowie eine Zunahme der Seneszenzmarker p16 und p21 [5]. Das Altern und die Lebensspanne des Türkisen Prachtgrundkärpflings lassen sich durch äußere Faktoren beeinflussen. Wird der Fisch durch vergrößerte Fütterungsintervalle einer Kalorienrestriktion unterzogen, so verlängert sich seine maximale Lebensspanne [6]. Erniedrigung der Temperatur oder Behandlung mit Resveratrol, das wegen seines Vorkommens im Rotwein bekannt wurde, führten ebenfalls zur Verlängerung der Lebensspanne des Fisches [7, 8]. Interessanterweise führt die Kreuzung eines längerlebigen mit einem kurzlebigen Stamm zu einer intermediären Lebensspanne der Nachkommen [9]. Genloci, die die Lebensspanne beeinflussen, lassen sich mit solchen Kreuzungsexperimenten und Genkartierungen identifizieren. Neben genetischen Faktoren beeinflusst auch das Mikrobiom in Form der Darmflora die Lebensspanne des Killifisches. Werden Fische im Alter von neun Wochen mit dem Darminhalt von sechs Wochen alten Fischen gefüttert, führt dies zur „Verjüngung“, was sich in erhöhter lokomotorischer Aktivität und verlängerter Lebensspanne zeigt [10].

Ein Ziel der Alternsforschung ist es zunächst, Alterungsprozesse zu verstehen, um letztlich die Gesundheitsspanne zu verlängern. Viele degenerative Krankheiten sind stark altersassoziiert. Daher sind regenerative Strategien besonders in Bezug auf das Altern wichtig. Der Killifisch, der z. B. seine Schwanzflosse regenerieren kann, stellt ein Modell dar, um alterungsabhängige Änderungen in Regenerationsprozessen zu analysieren. Bei der Schwanzflossenregeneration können die Wiederherstellung und der Aufbau mehrerer Gewebe, wie Knochen, Blutgefäße, Nerven, Bindegewebe oder Epidermis, analysiert werden. In einer Studie wurden alten und jungen Fischen 50 Prozent der Schwanzflosse amputiert. Während acht Wochen alte Fische innerhalb von 27 Tagen 


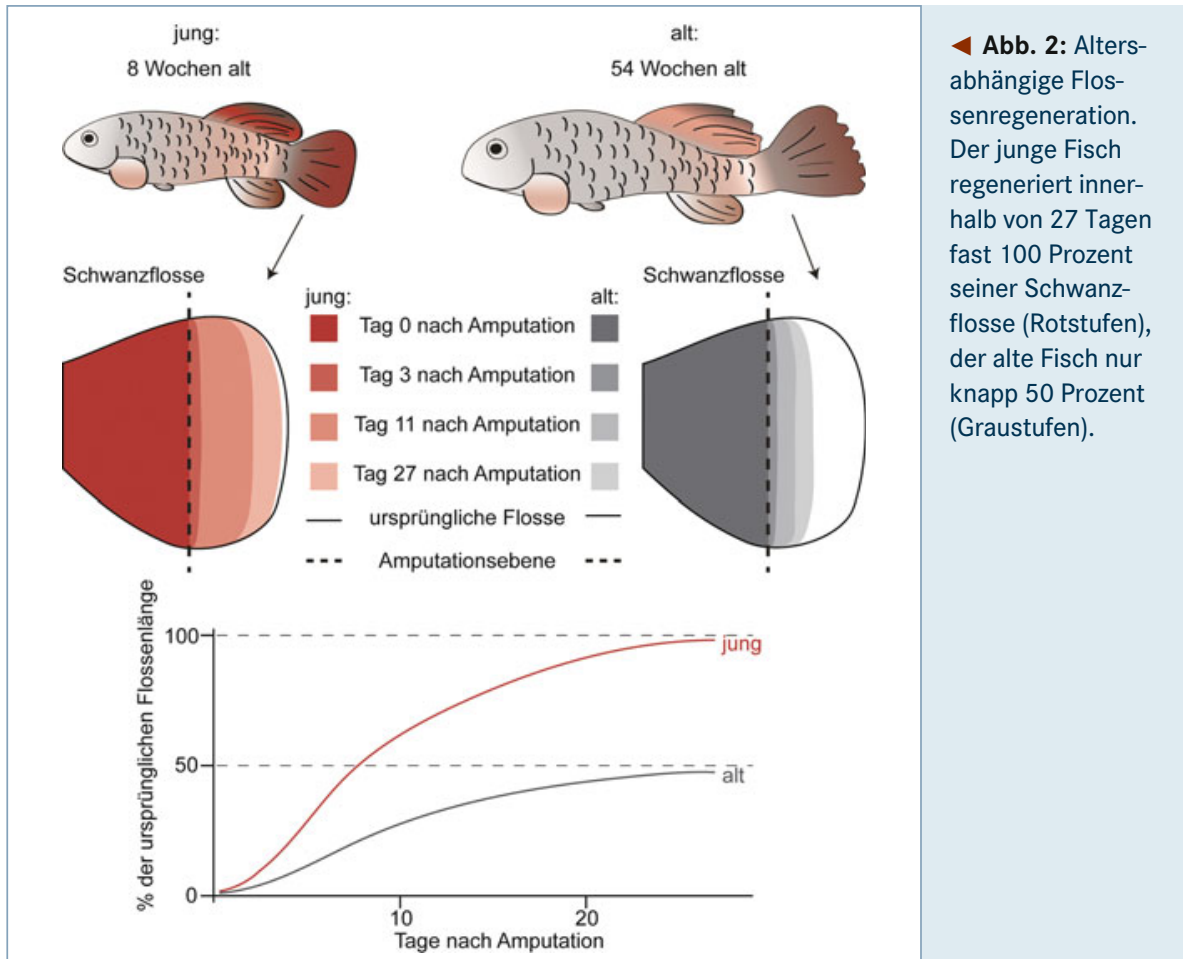

fast 100 Prozent ihrer Flosse regeneriert hatten, stellte die älteste Kohorte (54 Wochen alt) nur ca. 46 Prozent ihrer Schwanzflosse wieder her (Abb. 2). Die älteren Fische zeigten eine Verzögerung in der Knochenbildung, niedrigere Proliferationslevel und erhöhte Apoptoseraten. Auch Gene des Flossenregenerationsprogramms wurden in alten Fischen später aktiviert [11]. Dies liefert erste Ideen, wie sich zelluläre und molekulare Prozesse bei der Regeneration in jungen und alten Organismen unterscheiden, und öffnet Möglichkeiten, deren Regulation besser zu verstehen, um letztendlich zu regenerativen Therapien im Alter beizutragen.

\section{Genommanipulation beim Killifisch}

Um den Einfluss der Gene auf die Lebensspanne und das Altern zu verstehen, stellen Möglichkeiten zur genetischen Veränderung wertvolle Werkzeuge dar. Die Einbringung von Transgenen mithilfe der Transposase Tol2 durch direkte Injektion in befruchtete Eizellen des Prachtgrundkärpflings funktioniert sehr effizient [2]. Im Jahr 2015 wurde das Genom des Prachtgrundkärpflings publiziert [12, 13]. Mit ca. 1,5 Gigabasen ist es ungefähr halb so groß wie das menschliche Genom und in 18 Autosomenpaare sowie einem Paar Geschlechtschromosomen (XX oder XY) organisiert. Auf der Basis der Genomsequenz sind nun gezielte Veränderungen im Genom des Prachtgrundkärpflings möglich. So können mithilfe von CRISPR-Cas9 sowohl Gene ausgeschaltet als auch gezielte Sequenzveränderungen durchgeführt werden. Mithilfe dieser Technologie wurde unter anderem die Telomerase beim Killifisch inaktiviert, was zu einer Verkürzung der Telomerlänge von $\geq 6$ Kilobasen auf ca. 1,5 Kilobasen in Embryos der zweiten Generation führte [14]. Interessanterweise ist die Telomerlänge des Prachtgrundkärpflings der des Menschen sehr ähnlich. Dies steht im Gegensatz zur Maus, die etwa zehnmal längere Telomere besitzt, und unterstreicht, dass der Prachtgrundkärpfling hinsichtlich verschiedener Aspekte ein gutes Modell für das Altern des

\section{Literatur}

[1] Valdesalici S, Cellerino A (2003) Extremely short lifespan in the annual fish Nothobranchius furzeri. Proc Biol Sci 270 Suppl 2:S189-S191

[2] Platzer M, Englert C (2016) Nothobranchius furzeri: a model for aging research and more. Trends Genet 32:543-552 [3] Vrtílek M, Žak J, Pšenička M et al. (2018) Extremely rapid maturation of a wild, African annual fish. Curr Biol 28:R822R824

[4] Polacik M, Blazek R, Reichard M (2016) Laboratory breeding of the short-lived annual killifish Nothobranchius furzeri. Nat Protoc 11:1396-1413

[5] Graf M, Hartmann N, Reichwald K et al. (2013) Absence of replicative senescence in cultured cells from the short-lived killifish Nothobranchius furzeri. Exp Gerontol 48:17-28 [6] Terzibasi E, Lefrançois C, Domenici P et al. (2009) Effects of dietary restriction on mortality and age-related phenotypes in the short-lived fish Nothobranchius furzeri. Aging Cell 8:88-99

[7] Valenzano DR, Terzibasi E, Cattaneo A et al. (2006)

Temperature affects longevity and age-related locomotor and cognitive decay in the short-lived fish Nothobranchius furzeri. Aging Cell 5:275-278

[8] Valenzano DR, Terzibasi E, Genade T et al. (2006) Resveratrol prolongs lifespan and retards the onset of age-related markers in a short-lived vertebrate. Curr Biol 16:296-300 Menschen sein kann.
[9] Kirschner J, Weber D, Neuschl C et al. (2012) Mapping of quantitative trait loci controlling lifespan in the short-lived fish Nothobranchius furzeri - a new vertebrate model for age research. Aging Cell 11:252-261

[10] Smith P, Willemsen D, Popkes M et al. (2017) Regulation of life span by the gut microbiota in the short-lived African turquoise killifish. Elife 6:e27014

[11] Wendler S, Hartmann N, Hoppe B et al. (2015) Agedependent decline in fin regenerative capacity in the short lived fish Nothobranchius furzeri. Aging Cell 14:857-866 [12] Reichwald K, Petzold A, Koch P et al. (2015) Insights int sex chromosome evolution and aging from the genome of a short-lived fish. Cell 163:1527-1538

[13] Valenzano DR, Benayoun BA, Singh PP et al. (2015) The African turquoise killifish genome provides insight into evolution and genetic architecture of lifespan. Cell 163:1539-1554

[14] Harel I, Benayoun BA, Machado B et al. (2015) A platform for rapid exploration of aging and disease in a naturally short-lived vertebrate. Cell 160:1013-1026

Funding: Open Access funding provided by Projekt DEAL. Open Access: Dieser Artikel wird unter der Creative Commons Namensnennung 4.0 International Lizenz veröffentlicht, welche die Nutzung, Vervielfältigung, Bearbeitung, Verbreitung und Wiedergabe in jeglichem Medium und Form
erlaubt, sofern Sie den/die ursprünglichen Autor(en) und die Quelle ordnungsgemäß nennen, einen Link zur Creative Commons Lizenz beifügen un ordnungsgemä̈ nennen, einen Link zur Creative Commons Lizenz beifüg
angeben, ob Änderungen vorgenommen wurden. Die in diesem Artikel enthaltenen Bilder und sonstiges Drittmaterial unterliegen ebenfalls der genannten Creative Commons Lizenz, sofern sich aus der Abbildungslegende nichts anderes ergibt. Sofern das betreffende Material nicht unter der genannten Creative Commons Lizenz steht und die betreffende Handlung nicht nach gesetzlichen Vorschriften erlaubt ist, ist für die oben aufgeführten Weiterverwendungen des Materials die Einwilligung des jeweiligen Rechteinhabers einzuholen. Weitere Details zur Lizenz entnehmen Sie bitte der

Korrespondenzadresse:

Prof. Dr. Christoph Englert

Institut für Biochemie und Biophysik

Friedrich-Schiller-Universität Jena

Hans-Knöll-Straße 2

D-07745 Jena

c.englert@uni-jena.de

Leibniz-Institut für Alternsforschung -

Fritz-Lipmann-Institut (FLI)

Beutenbergstraße 11

D-07745 Jena

Christoph.englert@leibniz-fli.de www.leibniz-fli.de/de/forschung/ forschungsgruppen/englert/

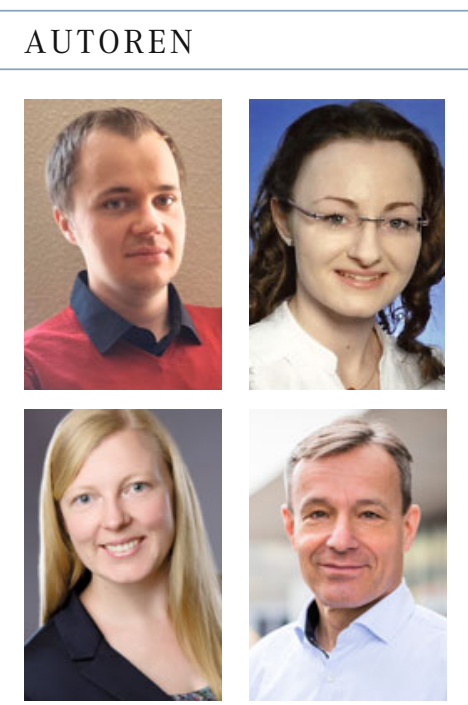

Johannes Krug, Annekatrin Richter (oben), Hanna Reuter und Christoph Englert (unten).

Die Arbeitsgruppe Molekulare Genetik untersucht anhand verschiedener Modellorganismen die genetischen Grundlagen des Alterns sowie von Regenerationsprozessen. 